\title{
Polysèmes
}

Revue d'études intertextuelles et intermédiales

\section{Passage et claustrophobie dans Voyage in the Dark de Jean Rhys}

\section{Sylvie Maurel}

\section{(2) OpenEdition}

\section{Journals}

Édition électronique

URL : http://journals.openedition.org/polysemes/1605

DOI : 10.4000/polysemes. 1605

ISSN : 2496-4212

Éditeur

SAIT

\section{Édition imprimée}

Date de publication : 1 janvier 2003

Pagination : 47-60

ISSN : 0999-4203

\section{Référence électronique}

Sylvie Maurel, "Passage et claustrophobie dans Voyage in the Dark de Jean Rhys », Polysèmes [En ligne], 6 | 2003, mis en ligne le 10 janvier 2017, consulté le 23 avril 2019. URL : http:// journals.openedition.org/polysemes/1605; DOI : 10.4000/polysemes.1605

Ce document a été généré automatiquement le 23 avril 2019

Polysèmes 


\title{
Passage et claustrophobie dans Voyage in the Dark de Jean Rhys
}

\author{
Sylvie Maurel
}

1 Deux types de passages sont à l'œuvre dans Voyage in the Dark ${ }^{1}$ : un passage transatlantique de l'île originaire à la métropole, en quête d'un avenir meilleur, et le passage à l'âge adulte, l'acquisition d'un nouveau statut, impliquant l'incorporation dans un complexe culturel dont Anna Morgan, jeune fille de dix-huit ans, originaire d'une île antillaise, doit maitriser les codes. Le voyage annoncé par le titre est à la fois une migration dans l'espace et un parcours cognitif, ce qui tend à désigner Voyage comme un roman d'apprentissage. Mais ce que nous murmure aussi ce titre, c'est que le parcours cognitif d'Anna promet de la mener à l'impasse. La formulation suggère à la fois ouverture et enfermement, mouvement et immobilité relative. Le « voyage » tourne court dès le second mot du titre, puisque la préposition «in ", là où peut-être on attendrait « into », si elle n'exclut pas le mouvement, le limite du moins à l'intérieur d'un même espace.

2 Voyage repose sur une opposition binaire, bipolarité que le titre original, Two Tunes ${ }^{2}$, programmait avec plus de netteté. Deux îles et leurs univers respectifs sont radicalement opposés dans ce texte qui s'attache à inscrire un ailleurs dans le cadre de la diégèse. Néanmoins, la machine textuelle évacue progressivement l'ailleurs figuré par l'île d'origine, pour mettre en place un monde claustral, un territoire de la claustrophobie, qui n'est pas sans évoquer la clôture tragique. Voyage travaille en effet à la création d'une fausse dialectique, ou de ce que Barthes appelle, à propos de la tragédie, « une dialectique impuissante $»^{3}$.

\section{Bipolarité...}

3 Si donc on peut parler de passage dans Voyage, c'est en partie qu'un ailleurs y est fortement représenté. Le récit oppose deux mondes, deux espaces: l'un est l'île paradisiaque où Anna a grandi, l'autre l'Angleterre où l'action se déroule. 
Même si la diégèse est exclusivement située en Angleterre, un «out there " vient sans cesse la dilater, dans pas moins de dix-sept analepses auxquelles il faut ajouter les nombreux moments où l'île natale est objet de discours, que ce soit dans les dialogues ou dans les monologues intérieurs qui nous ramènent sans cesse à ce paradis perdu. Sans relâche, le texte rejoue le passage qui conduisit Anna en Angleterre en passant lui-même d'un niveau de récit à l'autre. L'extradiégétique rivalise avec le diégétique : l'île natale resurgit à peu près à part égale avec le site du récit premier.

5 La matérialité de cet ailleurs sur lequel Anna porte un regard nostalgique est d'autant plus substantielle que l'île est très précisément située. Anna en donne la position, latitude et longitude à l'appui, par l'intermédiaire d'une citation :

'Lying between $15^{\circ} 10^{\prime}$ and $15^{\circ} 40^{\prime} \mathrm{N}$. and $61^{\circ} 14^{\prime}$ ' and $61^{\circ} 30^{\prime} \mathrm{W}$. A goodly island and something highland, but all overgrown with woods', that book said. (15)

6 Son existence étant attestée dans les livres savants, l'île se donne à lire comme un lieu réel, et le fait qu'elle ne soit jamais nommée l'investit en outre d'une existence fantasmée. Secteur de la carte géographique, elle est doublement substantielle en tant qu'elle s'inscrit aussi - surtout ? - comme la création d'une mémoire idéalisante.

7 Très substantiel, cet ailleurs qui conjugue plénitude sensorielle et réalité paroxystique ${ }^{4}$ est aussi radicalement autre. Il vient rivaliser avec la réalité diégétique à laquelle il s'oppose presque terme à terme, si l'on en croit, par exemple, la palette des couleurs :

The colours are red, purple, blue, gold, all shades of green. The colours here are

black, brown, grey, dim-green, pale blue, the white of people's faces-like

woodlice. (47)

On peut donc avoir l'impression que le texte travaille entre deux pôles et ménage ainsi la possibilité d'un passage. Tant que les deux espaces sont distincts, ils forment une alternative. En tant qu'espace euphorique, l'île originaire qui surgit ainsi aux côtés de l'île britannique opère à la manière d'un idéal que le texte expérimente comme manque, ce qui est absent mais ce vers quoi il reste tendu. La fonction de l'île natale, que son existence soit réelle ou fantasmée, est de créer un écart où le désir peut se déployer et vectoriser le parcours d'Anna. L'île originaire est à la fois l'espace du désir et celui du possible.

\section{...et fausse dialectique}

Peu à peu cependant, cette logique binaire et potentiellement dialectique est pervertie. La contradiction qui semble lier les deux espaces s'atténue, au détriment de l'ailleurs dont la représentation va en s'étiolant. Le texte évolue, semble-t-il, vers la fausse dialectique.

Dès l'incipit, l'île originaire se donne à lire comme un lieu en voie de disparition : « It was as if a curtain had fallen, hiding everything I had ever known. It was almost like being born again » (7). Le rideau tombe comme un couperet et sectionne, plus qu'il n'occulte, le passé ou l'ailleurs. Une nouvelle naissance a lieu: l'Angleterre ne sera donc pas simplement le site d'une expérience de la maturation; elle semble surgir du néant. Le rideau n'en demeure pas moins une ligne de démarcation instable et amovible, et, dans sa deuxième occurrence, la métaphore - en partie légitimée par la carrière qu'Anna embrasse en Angleterre - exprime davantage la porosité du barrage que la séparation: «[a] curtain fell and then I was here » (15). Malgré le «then », les deux procès semblent presque contemporains, comme si la syntaxe avait aboli l'espace de la traversée. Un pont 
syntaxique, assurant d'ailleurs le passage d'un niveau de récit à l'autre - cette phrase introduit une analepse - s'est substitué au passage géographique. Il n'y a plus de passage vers un probable ailleurs mais un mouvement oscillatoire, un va-et-vient entre imaginaire et réalité présentés comme interchangeables :

[s]ometimes it was as if I were back there and as if England were a dream. At other times England was the real thing and out there was the dream, but I could never fit them together. (7-8)

11 L'un des symptômes les plus probants de la disparition de l'alternative est le traitement que subit la figure du bateau dans Voyage. La traversée n'est pas représentée mais il est à plusieurs reprises question de bateau, quoique le plus souvent sous la forme d'une analogie. Invisible dans sa fonction de passeur, il apparait dans une comparaison : « [...] rows of little houses with chimneys like the funnels of dummy steamers and smoke the same colour as the sky » (8). Le paquebot s'est comme pétrifié ou sédentarisé. Il ressurgit plus loin dans un rêve que fait Anna, dans lequel elle essaie vainement de débarquer sur son île. Ce qui l'en empêche, c'est l'expansion surnaturelle du pont du bateau (140). Le navire est donc paradoxalement devenu le signe de l'impossibilité du passage ${ }^{5}$.

D'espace possible, l'ailleurs devient non-lieu. Conformément à son statut de métropole, il semble en effet que l'Angleterre ait la capacité d'absorber l'île natale, de la rayer de la carte. Monde insulaire et clos sur lui-même où les jardins sont "walled-in » (9) et où les rues forment des «shut-in ravines" (16), la métropole a tendance à abolir toute extériorité, comme un personnage annexe l'affirme : «"[i]t's a very nice place," she said, "so long as you don't suffer from claustrophobia" » (70). Et lorsqu'Anna quitte Londres pour Minehead où elle passe trois semaines, d'une part le texte choisit de ne pas représenter ce séjour, et, d'autre part, à son retour, Anna fait le constat suivant : «it's funny how it makes you feel that there's not anything else anywhere, that it's all made up that there is anything else » (78). La disparition de l'ailleurs fait place à l'incessant retour du même :

and the houses on either side of the street were small and dark and they were big and dark but all exactly alike. And I saw that all my life I had known that this was going to happen. (82)

Même les contraires («small» et "big») sont assimilables. Dès la fin de la première partie de Voyage, l'existence d'Anna tient davantage de la durée itérative que de la durée maturative. Après la rupture avec Jeffries en tout cas, le mouvement dialectique est devenu impossible. Les multiples déménagements d'Anna sont l'emblème de cette impossibilité. Si elle déménage six fois en moins de dix-huit mois, ces déménagements la conduisent de bedsit en bedsit qui se ressemblent tous, de même que ses tournées de chorus girl la conduisaient dans des villes toutes identiques. Anna circule mais ne va nulle part. Voyage est un roman du faux mouvement et de la claustrophobie.

\section{Espace tragique...}

Le roman d'apprentissage tourne alors à la tragédie, l'architexte tragique étant d'ailleurs clairement invoqué dans ce texte dont la toile de fond est éminemment théâtrale - Anna est « on the stage ». Lors de l'entrevue entre Anna et Hester, sa belle-mère, celle-ci, dotée d'un nom très racinien, décrit le sort du père d'Anna comme une tragédie : « [a]nd such a brilliant man poor man buried alive you might say yes it was a tragedy a tragedy » (53). Ailleurs, elle rappelle la tragédie de l'esclavage et la malédiction tragique qui pèse sur 
certaines familles de planteurs : " $[\mathrm{t}]$ he sins of the fathers Hester said are visited upon the children unto the third and fourth generation » (46). Bien entendu, dans la bouche d'Hester, le mot «tragedy" renvoie à une forme tragique dégradée: symptôme architextuel, il est aussi une métaphore usée.

La manière dont Barthes analyse la tragédie racinienne me paraît particulièrement apte à faire la lumière sur la «dialectique impuissante» de Voyage. Selon Barthes, l'espace tragique est ce qui fonde la tragédie racinienne : centrée autour d'un conflit fondamental entre deux êtres que lie un rapport d'autorité, la tragédie de Racine tient à ce que ces deux êtres sont enfermés dans un même lieu: "toute la tragédie semble tenir ", dit Barthes, "dans un vulgaire pas de place pour deux. Le conflit tragique est une crise d'espace $»^{6}$. Aux confins de la scène tragique, dans ce que Barthes appelle l'Anti-Chambre, s'ouvrent trois espaces extérieurs: l'espace de la mort, de la fuite et de l'événement. Ainsi, la mort physique n'appartient jamais à l'espace tragique en tant qu'elle est une réalité scandaleuse qui ne relève pas de l'ordre du langage, qui est le seul ordre tragique. La fuite, quant à elle, est en général recommandée par le personnel subalterne et non tragique de la tragédie. Celui-ci est chargé de mettre en évidence que la négation de la tragédie est toujours proche et facile, que le hors-scène est plein de solutions. Ces personnages secondaires sont également chargés de préserver le héros tragique du contact profane avec le réel, de lui épargner « la cuisine triviale du faire ». On aborde là la troisième fonction de l'espace extérieur, qui est de «tenir l'acte dans une sorte de quarantaine où ne peut pénétrer qu'une population neutre, chargée de trier les événements, d'extraire de chacun d'eux l'essence tragique et de n'apporter sur scène " que des nouvelles ou des récits, « des événements purifiés par le langage » .

Dans Voyage, l'Angleterre, cet univers qui se barricade, évoque l'enceinte tragique telle qu'elle est définie ici : elle maintient soigneusement hors de ses murs à la fois la mort, la fuite et l'événement. Pour ce qui est de la mort, Anna, qui est aussi la narratrice, ne quitte jamais la scène et l'avortement, quant à lui, a lieu dans les coulisses; n'en sont représentés que l'avant et l'après. La fuite, c'est-à-dire la solution dialectique, un temps possible ne serait-ce que dans l'imaginaire, est métaphoriquement exclue au travers de la figure du navire, on l'a vu. Après ce fameux rêve, les décrochages d'un niveau de récit à l'autre disparaissent. Il n'y a plus de référence à l'île d'origine ${ }^{8}$, si ce n'est dans la quatrième et dernière partie où tout se mêle dans le magma du courant de conscience, en un « montage a-chronologique $»^{9}$ qui pulvérise les structures spatio-temporelles.

17 Enfin, Voyage tient l'événement ou l'acte à l'écart. Le texte produit une figure emblématique de cette mise à distance, l'avortement. Sa fonction métaphorique est d'autant plus claire qu'Anna n'avorte pas pour les motifs que l'on attendrait. Ce n'est pas qu'elle est seule et n'a pas les moyens d'élever un enfant. C'est plutôt qu'elle a peur de mettre au monde un monstre (143), soit l'élément dissymétrique, l'exception, qui romprait la logique répétitive dans laquelle elle se trouve enfermée et qui n'est autre que la temporalité tragique :

[...] l'échec du héros racinien provient finalement d'une impuissance à concevoir le temps autrement que comme une répétition: l'alternative tend toujours à la répétition, et la répétition à l'échec. La durée racinienne n'est jamais maturative, elle est circulaire, elle additionne et ramène sans jamais rien transformer. ${ }^{10}$

La mise au monde d'un enfant, l'acte de naissance, ouvrirait une brèche dans le monde claustral d'Anna. Autant dire que l'événement constituerait une anomalie dans la durée itérative, "circulaire» de son existence. D'autres anomalies, d'autres alternatives 
susceptibles de mettre un terme à l'éternel retour du même se dessinent parfois, avant d'être immédiatement avortées par le texte. Il en va ainsi des débuts prometteurs de la liaison qu'Anna entretient avec Jeffries. Celui-ci lui envoie de l'argent et soudain tout devient possible : "[t]his is a beginning. Out of this warm room that smells of fur I'll go to all the lovely places I've ever dreamt of. This is a beginning [...] The streets looked different that day » (25). Ce geste a beau littéralement ouvrir des perspectives, l'ouverture s'avère très vite illusoire. L'ironie du sort veut qu'en acceptant l'argent de Jeffries, Anna devienne la " poule » que tous voient en elle, que ce soit sa logeuse - « "I don't want no tarts in my house, so now you know" » $(26)$ - ou sa belle-mère $(55,56)$. Inévitablement, l'alternative tend à la répétition, ce que le texte met particulièrement en évidence à la fin de la troisième partie, alors qu'Anna est allée voir la faiseuse d'anges et qu'elle a par conséquent lancé l'éradication de l'événement : « [e]verything was always so exactly alike -that was what I could never get used to. And the cold; and the houses all exactly alike, and the streets going north, south, east, west, all exactly alike » (152).

Donner lieu à l'événement, transformer la réalité, ne relève pas des compétences d'Anna. En cela, elle se rapproche du statut du héros tragique qui, comme le dit Barthes, « est l'homme du que faire? non du faire; il appelle, il invoque une action, il ne l'accomplit pas ; il pose des alternatives mais ne les résout pas $»^{11}$. Incapable d'agir, Anna formule à plusieurs reprises la nécessité de faire un projet :

of course, everything will be all right. I've only got to pull myself together and make a plan. (122)

I kept telling myself, 'You've got to think of something. You can't stay here. You've got to make a plan'. But instead I started counting all the towns I had been to, the first winter I was on tour [...]. (128)

For several days after that I kept on planning to leave London. The names of all the places I could go to went round and round in my head. (136)

Dans aucun des cas, le projet ne prend forme. Tantôt le texte s'arrête avant la description de son contenu, tantôt il représente l'informe en lieu et place de son élaboration: énumération mécanique ou circularité stérile se substituent à la formulation du plan d'action. Il s'agit là encore d'actes avortés, et le récit, soumis à cette logique répétitive, ne peut que devenir éminemment prévisible. Il se déploie tel une partition de Puccini, «the sort of music that you always know what's going to come next, that you can listen to ahead, as it were»(31), selon une temporalité proprement tragique, pour laquelle Barthes propose une formule très simple : « ce qui a été est $~^{12}$.

21 Ni la tragédie, ni Voyage ne représentent de parcours dialectique, et cela pour deux raisons. La première est que l'ordre tragique étant fondamentalement discursif, les mouvements du héros sont intransitifs, coupés du monde qui est maintenu hors scène. Selon Barthes,

le logos racinien ne se détache jamais de lui-même, il est expression, non transitivité, il n'introduit jamais au maniement d'un objet ou à la modification d'un fait. ${ }^{13}$

Privé de sa pragmatique, ou plus exactement l'ayant absorbée, le discours ne peut que se répéter, circuler dans une structure close et tautologique, « in the dark ». Anna parle et n'intervient pas sur son univers, elle ne parvient pas à lui imposer sa médiation. À la fin du roman, alors qu'elle réchappe à une hémorrhagie, il ne lui reste plus qu'à recommencer du début, comme le constate un médecin quelque peu désabusé : « "[s]he'll be all right," he said. "Ready to start all over again in no time, I've no doubt" (159). Voyage participe donc d'une " plastique de l'immédiation $»^{14}$. 

pas placée devant un choix impossible; elle se laisse porter. Son inertie, maintes fois soulignée, apparait plutôt comme une dégradation de l'immobilité tragique. Il n'y a pas de dilemme chez elle. À peine trouve-t-on dans le texte une parodie de monologue tragique, où s'exprime traditionnellement la division du héros : «I argued it out with myself quite solemnly, whether it was imagination or not that people are cruel » (126). La première fois que Jeffries lui donne de l'argent par exemple, ce qui fait d'elle une prostituée ou presque, Anna veut l'en empêcher mais ne le fait pas : «I meant to say, "What are you doing?" But when I went up to him instead of saying, "Don't do that," I said, "All right, if you like-anything you like, any way you like". And I kissed his hand " (33-34). La même aptitude à être agie apparaît alors qu'elle s'apprête à coucher avec Carl: «[w]hen he touched me I knew that he was quite sure I would. I thought, "All right then, I will” » (132). Elle se plie à la volonté de l'autre qui n'a même pas besoin d'être exprimée. Au total, il manque à Anna la stature de l'héroïne tragique.

On peut dès lors se demander si la disparition de l'île natale ou de l'ailleurs ne serait pas la métaphore de l'impossible accès à la scène tragique. Revenons à l'image du rideau. Lorsqu'Anna arrive en Angleterre, le rideau tombe au lieu de s'ouvrir ; il vient masquer la 
scène au lieu de la révéler. Associée à une arrivée, la métaphore est donc quelque peu paradoxale et nous livre peut-être une indication métatextuelle. Anna, qui flirte avec les planches du music-hall, flirte aussi avec celles du mélodrame. La scène tragique est quant à elle inaccessible.

L'̂le originaire est en fait le véritable espace tragique qui se refuse à Anna. «Les grands lieux tragiques ", dit Barthes, " sont des terres arides, resserrées entre la mer et le désert, l'ombre et le soleil portés à l'état absolu $»^{17}$. L'île natale répond tout à fait à ces critères, que ce soit selon Hester, celle qui introduit l'architexte de la tragédie dans Voyage - « nothing but rocks and stones and heat » (54) - ou selon Anna : « [i]t had a barren look, that place, a hot, frowning, barren look [...].The sky was terribly blue and very close to the earth » (62-63). C'est en Angleterre qu'Anna souffre de claustrophobie, mais l'île natale n'a, semble-t-il, rien à envier à la métropole en matière de clôture. Elle constitue un espace éminemment contraint, propre à donner lieu à la tragédie. De Morgan's Rest, le domaine familial, Anna dit par exemple : «[i]t was shut-in there, between two hills, like the end of the world [...]» (59).

Privée de cet espace tragique, Anna se retrouve prisonnière d'un espace mélodramatique dans lequel elle ne peut davantage intervenir. Ployant sous le poids d'un réel «idiot ", pour reprendre la terminologie de Clément Rosset, un réel insignifiant en tant qu'il n'est " susceptible d'aucune modification $»^{18}$, Anna est condamnée à déambuler « in the dark » sans pour autant prétendre à la dignité de l'héroïne tragique.

Malgré la bipolarité qui semble structurer Voyage et faire de la notion de passage une sorte de présupposé, tout concourt dans le texte à représenter l'impossibilité de tout mouvement dialectique. Cette " dialectique impuissante » s'explique, en partie du moins, par la tentation de la tragédie, genre qui finit lui-même par s'inscrire dans le texte comme l'alternative impossible. Voyage est en définitive le récit de l'impossible passage à la tragédie.

\section{NOTES}

1. Jean Rhys, Voyage in the Dark (1934), Harmondsworth: Penguin, 1969. Les numéros de page entre parenthèses renverront désormais à cette édition.

2. Voir Jean Rhys, Letters 1931-1966, Harmondsworth: Penguin, 1984, 149.

3. Roland Barthes, Sur Racine (1960), Paris : Éditions du Seuil, 1963, 31.

4. L'incipit, par exemple, énumère les senteurs extraordinairement variées de l'île natale. Un autre passage décrit Francine, nounou et confidente d'Anna, en train de déguster une mangue (58). Le texte décompose le geste pour mieux traduire la jubilation des sens.

5. On trouve une autre allusion à la traversée, avec à peu près les mêmes effets de sens, dans une lettre adressée à Hester, la belle-mère d'Anna : «now you write this extraordinary letter telling me [...] that you are willing to pay half her passage out here. Half her passage. But where's the other half coming from?»(52). L'étendue de l'océan n'est plus un lieu de passage mais un gouffre infranchissable.

6. Roland Barthes, op. cit, 30. 
7. Ibid., 11-13.

8. En fait il y en a une, brève, qui annonce le contenu de la fin du roman : «[1]ike that time at home with Meta, when it was Masquerade and she came to see me and put out her tongue at me through the slit in her mask» (151).

9. L'expression est due à Dorrit Cohn. Voir La Transparence intérieure (1978), Paris : Éditions du Seuil, 1981, 250.

10. Roland Barthes, op. cit., 52.

11. Ibid., 51.

12. Roland Barthes, op. cit., 42. La formule est d'ailleurs très proche de celle que Jean Rhys emploie dans sa correspondance pour expliquer ce qui se trame dans Voyage, alors en cours d'écriture : « $[t]$ he big idea-well I'm blowed if I can be sure what it is. Something to do with time being an illusion I think. I mean that the past exists-side by side with the present, not behind it; that what was-is » (Jean Rhys, Letters, op. cit., 24).

13. Roland Barthes, op. cit., 59.

14. Ibid, 47.

15. Ibid, 46.

16. Ibid., 31.

17. Ibid., 9.

18. Clément Rosset, Le Réel : Traité de l'idiotie, Paris : Éditions de Minuit, 1977, 20.

INDEX

oeuvrecitee Voyage in the Dark 